\title{
Formulación de metoclopramida en partículas poliméricas mucoadhesivas para administración intranasal
}

\author{
Marcela Vargas-Fresneda ${ }^{1}$, Flora A. Ganem² y Rubén J. Camargo ${ }^{1}$ \\ (1) Grupo de Investigación en Fisicoquímica de Bio y Nanomateriales, Facultad de Ingeniería, Universidad del Valle, \\ Cali - Colombia. (correo-e: marcela.vargas@correounivalle.edu.co; ruben.camargo@correounivalle.edu.co) \\ (2) Laboratorio de Investigación y Posgrado en Tecnología Farmacéutica, UNAM, FES-Cuautitlán, Cuautitlán Izcalli - \\ México. (correo-e: ganemq@hotmail.com)
}

Recibido Jul. 23, 2019; Aceptado Sep. 23, 2019; Versión final Nov. 12, 2019, Publicado Abr. 2020

\begin{abstract}
Resumen
Se prepararon y liofilizaron partículas (Ps) poliméricas mucoadhesivas a base de Gantrez®, cargadas con fármaco, mediante el método de desplazamiento de disolvente modificado, para administración intranasal de metoclopramida $\mathrm{HCl}(\mathrm{MCP})$. Las Ps obtenidas se caracterizaron midiendo tamaño $(368,0 \pm 79,6)$, índice de polidispersión $(0,294 \pm 0,014)$ y potencial Z $(-47,3 \pm 4,4)$ (Nanosizer, Malvern, ZS90). La carga de fármaco $(\% \mathrm{CF}=12,86 \pm 0,7)$ y la eficiencia de encapsulamiento $(\% \mathrm{EE}=77,14 \pm 3,9)$ se determinaron por un método espectrofotométrico UV, previamente validado. Se hizo un estudio de liberación in vitro, bajo condiciones sink, usando membranas de diálisis y un agitador orbital. Las partículas obtenidas poseen características tecnológicas adecuadas en cuanto a tamaño, contenido de fármaco y perfil de liberación, por lo que se consideran potencialmente adecuadas para la administración nasal de metoclopramida.
\end{abstract}

\section{Metoclopramide formulation into mucoadhesive polymeric particles for intranasal delivery}

\begin{abstract}
Using the modified solvent displacement method, drug-loaded mucoadhesive polymeric particles (Ps) based on Gantrez $\AA$, were prepared and freeze-dried, intended for intranasal delivery of metoclopramide $\mathrm{HCl}(\mathrm{MCP})$. Ps characterization was performed by measuring their size $(368,0 \pm 79,6)$, the polydispersity index $(0,294 \pm$ $0,014)$ and the Zeta potential $(-47,3 \pm 4,4)$ (Nanosizer, Malvern, ZS90). Drug loading $(\% \mathrm{DL}=12,86)$ and encapsulation efficiency $(\% E E=77,14)$ were determined by a UV spectrophotometric method (previously validated). In vitro release studies were carried out under sink conditions, using dialysis tubes and orbital shaker. The obtained particles possess attractive technological characteristics in terms of size, drug content and delivering profile, being potentially suitable for nasal administration of metoclopramide.
\end{abstract}




\section{INTRODUCCIÓN}

El clorhidrato de metoclopramida (MCP) es un potente antiemético, efectivo en el tratamiento de náusea y vómito asociado con quimioterapia y cáncer (Basante-Romo et al., 2016; López et al., 2013), embarazo, migraña y otros. Sin embargo, la biodisponibilidad de MCP es muy variable registrando valores entre 32 y $98 \%$ debido al metabolismo de primer paso en administración oral. A menudo los comprimidos de este fármaco son vomitados antes que la absorción sistémica ocurra, lo que obliga a la administración parenteral o rectal, en donde ambos métodos dan como resultado un ajuste deficiente del paciente al tratamiento. (Zaki et al., 2007). La MCP es un profiláctico que no sólo previene el vómito asociados con la quimioterapia del cáncer, sino que mejora los síntomas asociados con la gastroparesia diabética aguda y crónica y también se usa como terapia adyuvante en los pacientes con reflujo gastroesofágico. El uso de MCP se ve limitado por dos factores: un índice terapéutico estrecho y la taquifilaxia, que usualmente aparece dentro de las 6 semanas. Por su corta vida media (3-4 h) debe ser administrada vía oral 3 o 4 veces diarias, aunque para el caso de emésis agudas, se administra por vía intravenosa.

Para superar los principales inconvenientes de la vía oral y aumentar el ajuste del paciente al tratamiento, se buscan formas farmacéuticas y vías de administración alternativas a las convencionales (i.e., oral y parenteral). La fisiología de la mucosa nasal ofrece numerosas ventajas debido a la gran área superficial, la irrigación elevada y la presencia de linfocitos y mastocitos, la administración intranasal ha sido capitalizada para administración de fármacos de acción local y sistémica, empleando diferentes productos y dispositivos. Adicionalmente esta ruta es mínimamente invasiva, no provoca dolor y es de fácil manejo por parte del paciente (Sosnik et al., 2014). Sin embargo, el aclaramiento mucociliar propio de esta región del organismo aparece como un inconveniente para lograr que la formulación que contiene el fármaco logre un efecto terapéutico. Illum (2002), plantea que las formulaciones líquidas o en polvo que no son mucoadhesivas, tienen una vida media de 15 a 20 minutos antes de ser eliminadas por el aclaramiento mucociliar. Algunos autores resaltan la importancia del uso de un agente mucoadhesivo para mejorar los tiempos de residencia y en consecuencia, mejorar la biodisponibilidad (Irache et al., 2005; Pires y Santos, 2018). Por esto el uso de un polímero mucoadhesivo, como el copolímero de poli-vinil metil éter -co -anhídrido maléico (PVM/MA), es apropiado. Este copolímero, conocido comercialmente como Gantrez AN119 ${ }^{\circledR}$, es biocompatible y de baja toxicidad, por lo cual es ampliamente utilizado como adhesivo de prótesis dentales, parches y otros (Irache et al., 2005). Las partículas a base de este polímero se retienen en la mucosa por periodos de tiempo más prolongados que las de otros polímeros (Arbos et al., 2002). El objetivo del presente estudio fue preparar y caracterizar partículas poliméricas mucoadhesivas cargadas con MCP, que se adhieran a la mucosa nasal y liberen el fármaco para lograr un efecto sistémico.

\section{METODOLOGÍA}

Se presentan los materiales y métodos empleados para la preparación y caracterización de las partículas.

\section{Materiales}

Gantrez AN119 ${ }^{\circledR}$ donado por Ashland Specialty Ingredients, México; acetona (Tecsiquim SA de CV, México); clorhidrato de metoclopramida micronizada (IPCA Laboratories Limited. Madhya, Pradesh, India) donado por Moléculas Finas de México, México. Para las pruebas de liberación se usaron membranas de diálisis de tamaño de poro MWCO: 6-8 kD, de $50 \mathrm{~mm}$ de ancho y $70 \mathrm{~mm}$ de largo (Spectrum Labs, Inc., USA) e hilo de nylon para sujetarlas en los extremos. Agua destilada se obtuvo de un sistema Milli-Q (Millipore, Schwalbach, Germany). Hidróxido de sodio (perlas) y fosfato de potasio monobásico (Mallinckrodt Baker, S.A. de C.V., México). Todos los reactivos fueron usados sin purificación adicional. La estructura química de la metoclopramida se muestra en la figura 1.<smiles>CCN(CC)CCNC(=O)c1cc(Cl)c(N)cc1OC</smiles>

Fig. 1. Estructura química de la metoclopramida. (Zaki et al., 2007) 


\section{Preparación de las partículas}

Mediante el método de desplazamiento de disolvente modificado (Figura 2), se prepararon partículas (Ps) poliméricas mucoadhesivas de dos tipos: placebo (Ps-pcb) y cargadas con metoclopramida (Ps-MCP) a diferentes proporciones fármaco:polímero (Tabla 1). Para ello se pesaron $110 \mathrm{mg}$ de Gantrez ${ }^{\circledR}$ disueltos en 5 $\mathrm{mL}$ de acetona que posteriormente se inyectaron en $20 \mathrm{~mL}$ de agua, la inyección fue manual en el seno del líquido a $\mathrm{pH}=12$, se usó $\mathrm{NaOH}$ para ajustar el $\mathrm{pH}$. Esta mezcla se mantuvo bajo agitación por 30 minutos a $5000 \mathrm{rpm}$ (Ultraturrax, IKA T18, Alemania). Para el caso de las Ps cargadas con fármaco, éste se disolvió junto con el polímero en acetona, a 10, 15, 20 y $30 \%$ en peso de fármaco respecto al peso de polímero. Durante la inyección, la difusión de la acetona en la fase acuosa resulta en la formación de las partículas por precipitación (Arbos et al., 2002). Una vez formadas las partículas, se removió la fase orgánica de la dispersión al vacío y calentando a $40^{\circ} \mathrm{C}$ por 10 minutos (Rotavapor, BUCHI R3, Alemania). El resultante se separó en centrífuga a $18000 \mathrm{rpm}$ durante 10 minutos (Centrífuga 5804, Eppendorf, Alemania). El sobrenadante y el precipitado fueron colectados en recipientes herméticos. El sobrenadante se congeló a $-20^{\circ} \mathrm{C}$ durante 20 horas aproximadamente y finalmente se liofilizó durante 24 horas, sin crioprotector, a 0,600 mBar y $-50^{\circ} \mathrm{C}$ (Liofilizadora FreeZone 2.5 Labconco, USA). Para la preparación de las Ps-pcb se siguió el mismo procedimiento sin MCP.

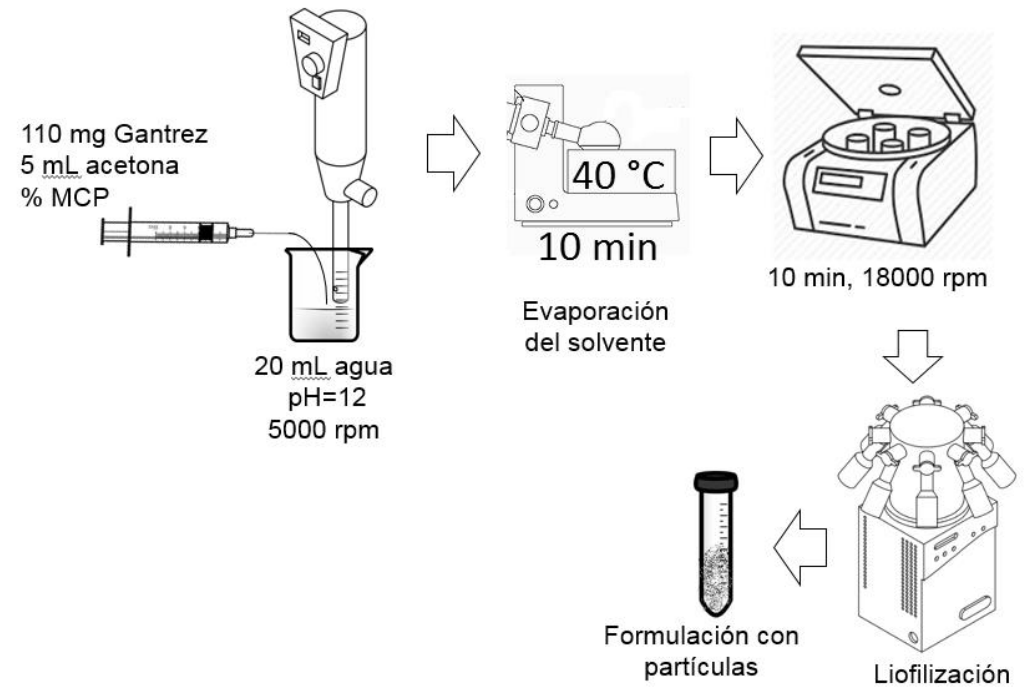

Fig. 2. Método de desplazamiento de disolvente modificado utilizado para la preparación de las Ps

Tabla 1. Proporciones fármaco:polímero empleadas para preparar las Ps

\begin{tabular}{|c|c|}
\hline Código de formulación & Proporción MCP:polímero \\
\hline Ps pcb & $0: 1$ \\
\hline Ps-10MCP & $0,10: 1$ \\
\hline Ps-15MCP & $0,15: 1$ \\
\hline Ps-20MCP & $0,20: 1$ \\
\hline Ps-30MCP & $0,30: 1$ \\
\hline
\end{tabular}

\section{Caracterización fisicoquímica de las partículas}

Se prepararon las formulaciones según la metodología descrita y las partículas obtenidas se caracterizaron midiendo tamaño, índice de polidispersión y potencial Z (Zetasizer ZS90, Malvern, UK) por la técnica de dispersión de luz dinámica (DLS: dynamic light scattering) y Electroforésis Doppler con Láser. Las medidas se hicieron por triplicado, en todas las formulaciones preparadas. La mucoadhesión se evaluó de forma indirecta en un trabajo previo, determinando la interacción de las partículas con mucina, empleando ácido bicinconínico para cuantificar la mucina libre (Aguilar et al., 2014). La eficiencia de encapsulación y la carga de fármaco en las partículas se determinaron por espectrofotometría UV, mediante un método validado parcialmente, para el cual se utilizó un estándar USP de clorhidrato de metoclopramida (MCP).

También se realizó calorimetría diferencial de barrido (TGA/DSC 1, STARe System, Mettler Toledo, USA) a los componentes por separado, a la mezcla y a las partículas cargadas. Se usaron de 3 a 4 miligramos de muestra dispersa uniformemente en el fondo de un crisol estándar de aluminio de $100 \mu \mathrm{m}$ de capacidad con tapa, usando un crisol vacío con tapa como referencia. La medida se llevó a cabo en atmósfera de nitrógeno con flujo constante $50 \mathrm{~mL} / \mathrm{min}$, a una velocidad de calentamiento de $10^{\circ} \mathrm{C} / \mathrm{min}$ en un rango de $25^{\circ} \mathrm{C}$ hasta 
$280^{\circ} \mathrm{C}$, con enfriamiento. Se reportó el rango de $100^{\circ} \mathrm{C}$ a $280^{\circ} \mathrm{C}$ pues es donde tienen lugar las transiciones de interés, antes de $100^{\circ} \mathrm{C}$ sólo se observa la pérdida de agua superficial. El enfriamiento tuvo como propósito retirar el agua adsorbida. (Salinas et al., 2011).

\section{Ensayo de liberación del fármaco a partir de la dispersión de partículas}

La liberación del fármaco se monitoreó in vitro usando membranas de diálisis MWCO: 6-8 kD, de $50 \mathrm{~mm}$ de ancho y $70 \mathrm{~mm}$ de largo (Spectrum Labs, Inc., USA), conteniendo $1 \mathrm{~mL}$ de disolución de partículas cargadas. Para cada bolsa se hicieron pesadas independientes, de $8 \mathrm{mg}$ de formulación liofilizada, lo cual equivale a 1 $\mathrm{mg}$ de MCP, aproximadamente. Como medio de liberación, se empleó buffer fosfato a un pH de 6,6, valor similar al pH de la mucosa nasal humana (Salade et al., 2019; Sosnik et al., 2014). Con el propósito de mantener las condiciones sink, las bolsas formadas con la membrana (Figura 3), se sumergieron en $30 \mathrm{~mL}$ del medio de liberación, a $37^{\circ} \mathrm{C} \pm 1^{\circ} \mathrm{C}$, a $40 \mathrm{rpm}$, en un agitador orbital (Baño de agua, WNE14, Memmert, Alemania). Se tomaron alícuotas de $2 \mathrm{~mL}$ del medio de liberación a intervalos de 15, 30, 45 minutos, 1, 2, 3, $4,5,6,7,8,24$ horas y cada alícuota fue reemplazada con $2 \mathrm{~mL}$ de medio fresco, para asegurar que esté muy por debajo de la saturación del medio.

Las muestras se diluyeron y se midieron en un UV-Vis (HITACHI U 5100, Japón) a una longitud de onda de $308 \mathrm{~nm}$, característica de la metoclopramida (Valencia et al., 2011). La concentración del fármaco se determinó a partir de una curva de calibración construida previamente. Los experimentos de liberación se hicieron por triplicado usando Ps-pcb como blanco.

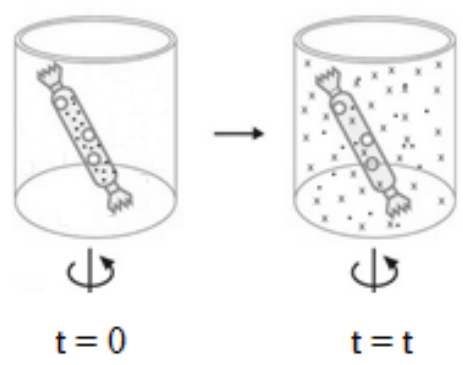

Fig. 3. Esquema de los viales para la prueba de liberación in vitro

Se calcularon los porcentajes de carga de fármaco (\%CF) y eficiencia de encapsulamiento (\%EE), los cuales están definidos por las ecuaciones (1) y (2), respectivamente (Chen et al., 2014). La preparación de las muestras para esta determinación, consiste en dejar una cantidad conocida de formulación en un medio acuoso, para que las partículas se hidrolicen por completo, posteriormente se centrifugan, se separa el sobrenadante y se lleva a un volumen de aforo, para leer en el espectrofotómetro UV. El porcentaje de carga de fármaco, está relacionado con la capacidad de carga, dado que considera la diferencia entre la cantidad inicial de fármaco añadido durante la preparación de las partículas y la cantidad de fármaco libre, es decir la concentración de fármaco que se obtiene en el sobrenadante después de centrifugar. (Butani et al., 2016). El porcentaje de eficiencia de encapsulamiento, da cuenta de la cantidad de fármaco que efectivamente se encuentra dentro de las partículas (Aguilar et al., 2014), relacionando la carga de fármaco observada o calculada, con la carga de fármaco teórica.

$$
\begin{aligned}
& \% \mathrm{CF}=\left(\frac{\text { Peso de MCP en las Ps }}{\text { Peso de Ps con MCP }}\right) \times 100 \\
& \% \mathrm{EE}=\left(\frac{\% \mathrm{CF} \text { calculado }}{\% \mathrm{CF} \text { teórico }}\right) \times 100
\end{aligned}
$$

\section{Validación del método analítico}

Se hizo la validación parcial del método analítico. Se encontró que la máxima longitud de onda fue de 308 $\mathrm{nm}$. El método exhibe alta sensibilidad con linealidad en el rango de 2 a $16 \mu \mathrm{g} / \mathrm{mL}$, todas las curvas de calibración presentaron una relación lineal entre la absorbancia y la concentración, con un coeficiente de correlación $R^{2}=0,9987$, con un coeficiente de variación $\leq 2,70 \%$. La curva de regresión fue $y=0,0355 x+$ 0,0071 . La precisión del método fue buena $(C V=2,71 \%)$. La solución de muestra tenía una estabilidad de 36 horas, protegida de la luz, en este tiempo no se encontró diferencia significativa de la concentración del analito $(p<0,05)$. El límite de detección (LOD) fue de $0,0131 \mu \mathrm{g} / \mathrm{mL}$ y el límite de cuantificación (LOQ) fue de 0,0398 $\mu \mathrm{g} / \mathrm{mL}$. (Armbruster y Pry, 2008) 


\section{RESULTADOS Y DISCUSIÓN}

Este apartado se divide en cuatro subsecciones: preparación de las partículas, caracterización fisicoquímica de las partículas, análisis calorimétrico y ensayo de liberación in vitro.

\section{Preparación de las partículas}

Por el método de desplazamiento de disolvente modificado se prepararon partículas poliméricas a base de Gantrez AN119 ${ }^{\circledR}$ y conteniendo metoclopramida (MCP). Tomando en consideración las propiedades del fármaco como pKa $(9,04 ; 14,49)$, fue necesario alcalinizar el agua de la preparación para tener la forma no ionizada del fármaco, y lograr así una mejor incorporación de éste en la matriz polimérica. Se usó $\mathrm{NaOH}$ hasta alcanzar un $\mathrm{pH}=12$. Considerando lo reportado por Salade et al. (2019), la mucosa nasal actúa como una barrera lipofílica, por lo cual la forma no ionizada de la metoclopramida tendrá un mejor transporte en la membrana. El agitador de alta velocidad (Ultraturrax) y el goteo de la solución de acetona, polímero y fármaco permitió obtener partículas con tamaños que variaron entre 229,0 y 560,7 nm. Para la liofilización se congelaron las muestras en tubos falcon, a $-20^{\circ} \mathrm{C}$, durante 20 horas aproximadamente. La presencia de etanol, estaba afectando el proceso de congelación de la muestra, al bajar el punto de congelación, por lo cual se decidió modificar el método de síntesis propuesto por Arbós y no usar etanol. Con el fin de evaluar la necesidad del uso de dextrosa como crioprotector, se hicieron liofilizaciones a 0,600 mBar y $-50^{\circ} \mathrm{C}$, con y sin dextrosa. Se midieron, por triplicado, los tamaños de las partículas antes y después de liofilizar. Se observó que la muestra liofilizada y resuspendida en agua Milli-Q, presentaba un incremento en el tamaño no superior al $2 \%$, es decir no se presentó agregación ni colapso de las partículas, por lo cual, se decidió prescindir del uso de crioprotector.

\section{Caracterización fisicoquímica de las partículas}

En la Tabla 2 se presentan los resultados de las pruebas de caracterización realizadas a las diferentes formulaciones de partículas. Para tamaño y potencial zeta, se presentan los resultados como media \pm desviación estándar, mientras que para el PDI, \%CF y \%EE como media $\pm I C$, con un intervalo de confianza (IC) al $95 \%$ y $n=3$ (Crucho y Barros, 2017). Las formulaciones exhiben un tamaño entre 229,0 y 560,7 nm, con un bajo índice de polidispersión (Pdl) (Camacho et al., 2011). El tamaño de partícula es un factor crítico que afecta su desempeño en la liberación de fármacos (Flores et al., 2017). Así, partículas menores a $1 \mu \mathrm{m}$ son más eficientes al penetrar hasta $25 \mu \mathrm{m}$ de profundidad a través de la barrera de la mucosa (González et al., 2017). Por otro lado, Butani y colaboradores (2016) afirman que un potencial zeta alto produce repulsión eléctrica y da estabilidad a la suspensión de Ps.

En la Tabla 2 se observa que el valor absoluto de potencial zeta de las diferentes formulaciones, fue mayor a $40 \mathrm{mV}$, un indicador de la estabilidad de las partículas de PVM/MA en suspensión acuosa (Chen et al., 2012). Al comparar el valor de potencial zeta de las partículas placebo con las demás formulaciones, se puede inferir que la metoclopramida (MCP) no tiene influencia en dicho parámetro. Tanto los porcentajes de carga de fármaco (\%CF) como la eficiencia de encapsulamiento (\%EE) aumentan, indicando que en la formulación se incrementa la cantidad de fármaco con respecto al polímero. El \% EE, aumenta alcanzándose un máximo en la proporción fármaco:polímero 0,20:1, sin embargo la formulación con proporción 0,30:1 tiene un \%CF mayor con un valor de \%EE cercano.

Tabla 2. Resultados de la caracterización de las formulaciones de Ps preparadas con diferentes proporciones MCP:polímero

\begin{tabular}{|c|c|c|c|c|c|c|}
\hline Formulación & $\begin{array}{c}\text { Proporción } \\
\text { MCP:polímero }\end{array}$ & Tamaño $(\mathrm{nm})$ & Pdl & $\begin{array}{c}\text { Potencial } Z \\
(\mathrm{mV})\end{array}$ & $\% C F$ & $\% E E$ \\
\hline Ps pcb & $0: 1$ & $229,0 \pm 65,8$ & $0,233 \pm 0,011$ & $-58,8 \pm 4,9$ & - & - \\
\hline Ps-10MCP & $0,10: 1$ & $368,0 \pm 79,6$ & $0,294 \pm 0,014$ & $-47,3 \pm 4,4$ & $8,03 \pm 0,4$ & $60,61 \pm 3,0$ \\
\hline Ps-15MCP & $0,15: 1$ & $445,5 \pm 82,1$ & $0,366 \pm 0,018$ & $-70 \pm 6,3$ & $8,13 \pm 0,4$ & $62,52 \pm 3,1$ \\
\hline Ps-20MCP & $0,20: 1$ & $489,0 \pm 87,8$ & $0,488 \pm 0,024$ & $-62,5 \pm 11,1$ & $12,86 \pm 0,7$ & $77,14 \pm 3,9$ \\
\hline Ps-30MCP & $0,30: 1$ & $560,7 \pm 104,3$ & $0,258 \pm 0,012$ & $-40,7 \pm 7,4$ & $16,38 \pm 0,8$ & $70,6 \pm 3,5$ \\
\hline
\end{tabular}

\section{Análisis calorimétrico}

Para el análisis calorimétrico, según lo propuesto por Crucho et al. (2017), sólo se observó lo ocurrido hasta los $280^{\circ} \mathrm{C}$, ya que la degradación del PVM/MA ocurre a $268^{\circ} \mathrm{C}$. Las transformaciones relevantes de la MCP pura tienen lugar a $150^{\circ} \mathrm{C}$, la fusión del fármaco y a $185^{\circ} \mathrm{C}$ la degradación de este (El-Habeeb et al., 2014). En el caso de la mezcla física de los componentes, se observa un desplazamiento de $10{ }^{\circ} \mathrm{C}$ respecto a la temperatura de degradación de la MCP. Cuando la MCP está mezclada físicamente requiere de una 
temperatura más alta para degradarse $\left(195^{\circ} \mathrm{C}\right)$, esto se debe a interacciones entre el polímero y el fármaco en la mezcla. Este ligero desplazamiento también se puede atribuir a que el Gantrez ${ }^{\circledR}(77 \% \mathrm{w} / \mathrm{w})$, tiene una temperatura de degradación superior a la del fármaco $\left(268{ }^{\circ} \mathrm{C}\right)$. En la figura 4 , se observa que el perfil correspondiente a partículas cargadas (Ps-30MCP) no presenta el evento endotérmico característico de la MCP en su estado cristalino, por lo que se puede concluir que el fármaco se encuentra disperso molecularmente en la matriz polimérica. Además, no se observaron cambios ni desplazamientos en la temperatura de transición vítrea $\left(\mathrm{Tg}=152^{\circ} \mathrm{C}\right)$ del Gantrez (Chung et al., 1990).

Después de la caracterización fisicoquímica de las nanopartículas preparadas se puede afirmar que el método de desplazamiento de disolvente modificado permite obtener partículas cargadas de fármaco, con propiedades mucoadhesivas que logren liberar el fármaco de manera controlada en la mucosa nasal y a través de la misma. La mucoadhesividad fue verificada por Aguilar (2014) en colaboración con Ganem, en un trabajo previo, quienes evaluaron la interacción de las NPs con mucina, determinando de manera indirecta la mucoadhesión de las partículas. Cerchiara et al. (2005) encontraron que formulaciones de partículas a base de PVM/MA se adhirieron apropiadamente a mucosa nasal de vaca y confirma lo encontrado por Aguilar et al. (2014). Resalta la superioridad de un sistema de liberación modificada mucoadhesivo frente a uno que no lo es (Sosnik et al., 2014). Esto al observar parámetros tales como la concentración máxima, tiempo para la concentración máxima, área bajo la curva después de $24 \mathrm{~h}$ y velocidad de eliminación. Un sistema mucoadhesivo ofrece ventajas en términos de liberación sostenida en administración intranasal (Sosnik et al., 2014).

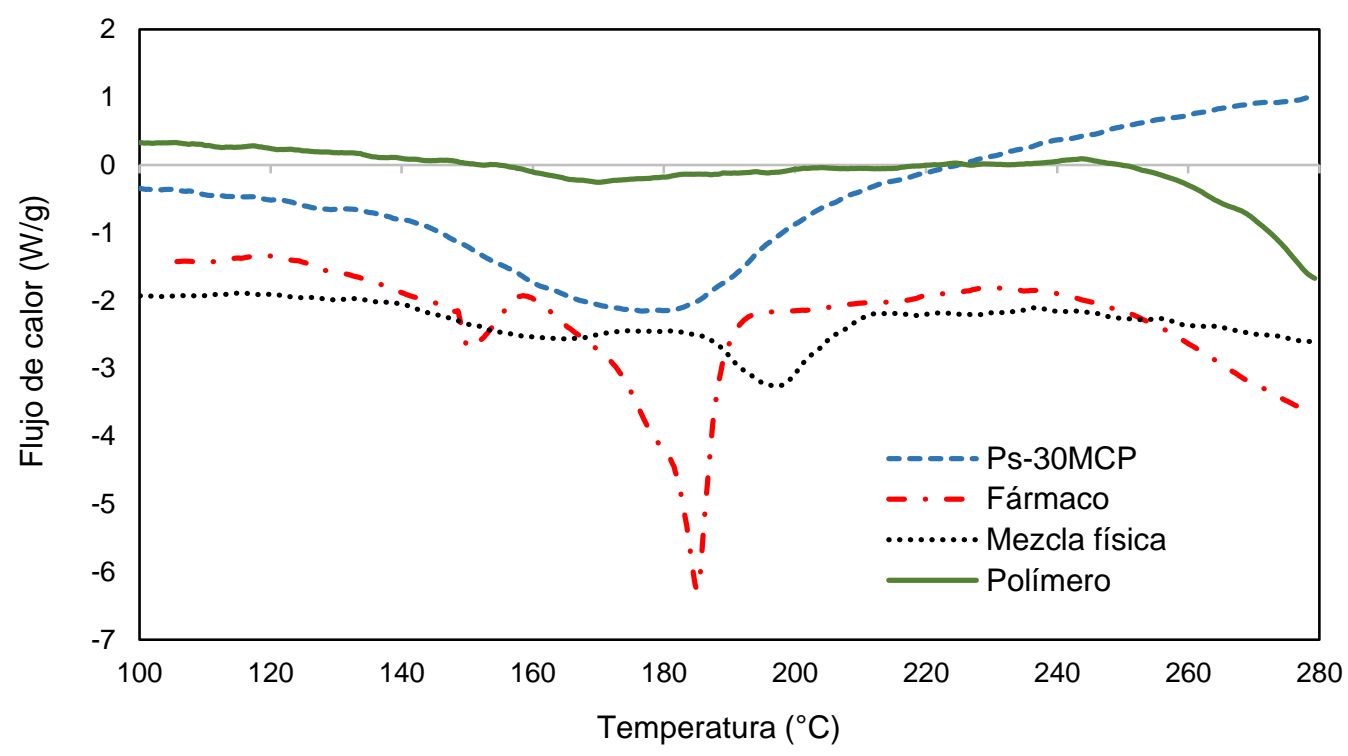

Fig. 4. Termogramas de partículas-Ps-30MCP $(P)$, fármaco $M C P(F)$, mezcla física de los componentes (MF) y polímero Gantrez ${ }^{\circledR}(\mathrm{P})$

\section{Ensayo de Liberación In Vitro}

La carga de fármaco y la eficiencia de encapsulamiento calculadas para cada formulación se presentaron en la Tabla 2, donde se aprecia que las formulaciones con mayor carga de fármaco son las de $20 \%$ y $30 \%$ de metoclopramida con respecto al peso de polímero agregado. Estas formulaciones se eligieron para llevar a cabo el ensayo de liberación in vitro, cuyo perfil se muestra en la Figura 5. Los resultados obtenidos señalan a Ps-30MCP, la formulación con $30 \%$ de metoclopramida, como la mayor carga de MCP para ser utilizada (\%CF y \%EE). La liberación prolongada de la metoclopramida desde las partículas de PVM/MA puede atribuirse a la biodegradabilidad del polímero. Se reporta la existencia de cinco mecanismos de liberación de fármacos a partir de partículas: (i) desorción del fármaco ligado a la superficie, (ii) difusión a través de la matriz polimérica, (iii) difusión a través de la pared polimérica de cápsulas, (iv) erosión de la matriz de la partícula y (v) un proceso combinado de erosión-difusión (Kumari et al., 2010). En los perfiles de liberación (Figura 5 y Figura 6), se observa una liberación inicial rápida o "tipo ráfaga" atribuible al fármaco que se encuentra adsorbido en la superficie hidroxilada de las partículas de Gantrez ${ }^{\circledR}$ (Camacho et al., 2011).

Tas y colaboradores, evaluaron la permeabilidad de la metoclopramida contenida en diferentes formulaciones (solución, gel y polvo) y encontraron que el polímero y la conformación del mismo, tienen un efecto sobre el porcentaje de liberación del fármaco, es decir un alto contenido de polímero, conformado en micelas va a dar 
lugar a un sistema muy intrincado que dificulta la difusión del fármaco, porque reduce el área de difusión molecular efectiva, lo cual permite afirmar que una formulación de partículas puede ser superior en desempeño a los sistemas de gel o hidrogel. (Tas et al., 2009)

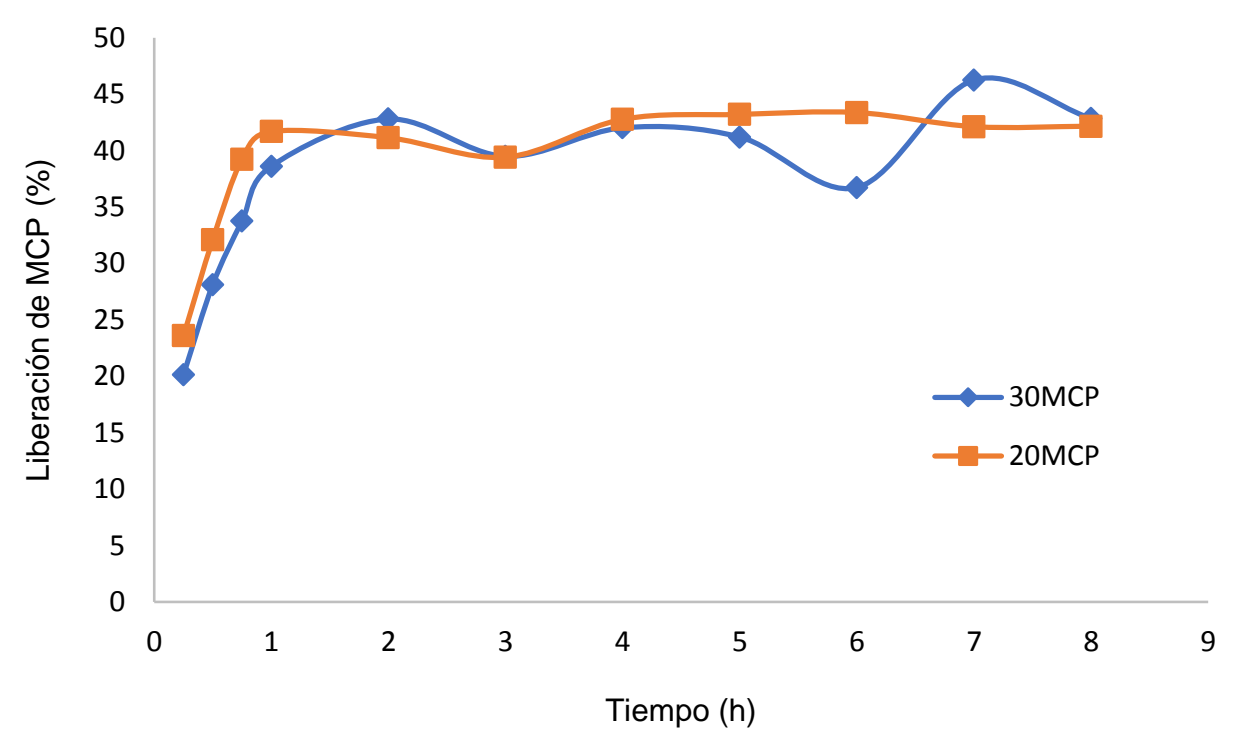

Fig. 5. Perfil de liberación de las Ps con $20 \%$ y $30 \%$ de fármaco

En la Tabla 3 se puede observar el contenido de fármaco, el porcentaje liberación de fármaco a 24 horas y la cantidad de formulación necesaria para una dosis terapéutica a 24 horas de $0,5 \mathrm{mg} \mathrm{MCP} / \mathrm{Kg}$, esto para formulaciones de Ps-20MCP y Ps-30 MCP. Del análisis de los datos, se puede observar como la formulación de partículas Ps-20MCP, aunque tiene menos carga inicial (\%CF) de MCP, es la formulación que necesita menor cantidad para completar una dosis terapéutica a 24 horas. La explicación está en que esta formulación tiene una capacidad un poco más alta de liberar el fármaco MCP y mayores polidispersión Pdl y capacidad de encapsulamiento \%EE (Tabla 2).

En ensayos preliminares donde el medio receptor se agitaba empleando una barra magnética a una velocidad aproximada de $700 \mathrm{rpm}$, se observó que se liberaba aproximadamente el $95 \%$ a las $8 \mathrm{~h}$ y a las $24 \mathrm{~h}$, se tenía en promedio un $98 \%$ liberado. No obstante, considerando las características de la mucosa nasal y que a pesar de que se tiene un movimiento ciliar que tiende a arrastrar el moco y todos los fluidos secretados, junto con los cuerpos extraños que ingresen, en realidad el movimiento no es tan intenso, por lo que se decidió realizar los estudios de liberación con una agitación más moderada de 40 rpm, empleando un agitador orbital (Baño de agua, WNE14, Memmert, Alemania). Factores como el tipo de agitación, la velocidad de agitación y el volumen de medio receptor impactan de manera directa en la cantidad liberada y en la velocidad de liberación. Algo importante a tomar en cuenta es que la dosis requerida de formulación para conseguir un efecto terapéutico es muy pequeña, aun pensando que no se libera el total de fármaco incluido en las nanopartículas.

Tabla 3. Contenido y liberación de MCP, miligramos de formulación para dosis terapéutica a 24 horas

\begin{tabular}{|l|c|c|c|c|}
\hline Formulación & $\begin{array}{c}\mathrm{mg} \mathrm{MCP} / \mathrm{mg} \\
\text { formulación }\end{array}$ & $\begin{array}{c}\text { \% de liberación } \\
\text { de MCP a 24h }\end{array}$ & $\begin{array}{c}\text { (mg MCP liberados a } \\
24 \mathrm{~h}) /(\mathrm{mg} \text { formulación) }\end{array}$ & $\begin{array}{c}\text { mg de formulación para dosis } \\
\text { terapéutica a 24h de 0,5 mg MCP/Kg }\end{array}$ \\
\hline Ps-20MCP & 0,129 & 89,4 & 0,115 & 4,3 \\
\hline Ps-30MCP & 0,164 & 42,5 & 0,070 & 7,2 \\
\hline
\end{tabular}

Con la idea de describir la cinética del proceso de liberación de las formulaciones con $20 \%$ y $30 \%$ de fármaco, se usaron los datos de porcentaje de liberación registrados durante las primeras dos horas, para determinar si se ajustaban a las ecuaciones del modelo matemático simplificado de Higuchi, Ley de potencia (Ecuación de Korsmeyer-Peppas), cinética de orden cero y cinética de primer orden. Se escogieron los datos de las dos primeras horas, porque en la figura 5 se observa que después de este tiempo ya no se libera más fármaco, se tiene prácticamente una asíntota. Las ecuaciones utilizadas para estos modelos fueron las siguientes (Costa y Sousa Lobo, 2001; Paul, 2011; Petropoulos et al., 2012; Rezaeinia et al., 2019; Wu et al., 2019): 


\section{Modelo de Higuchi}

El modelo más simple propuesto originalmente por Higuchi está dado por la siguiente ecuación:

$$
M t=[D C s(C-C s) t]^{1 / 2}
$$

Donde, Mt es la cantidad de fármaco liberada al tiempo t por unidad de área, $C$ es la concentración inicial de fármaco, Cs es la solubilidad del fármaco en la matriz y $D$ es el coeficiente de difusión del fármaco en la matriz que lo contiene. La ecuación simplificada del modelo de Higuchi es:

$$
M t=K_{H} t^{1 / 2}
$$

Siendo $K_{H}$ la constante de disolución de Higuchi. El modelo de Higuchi es válido durante todo el tiempo de liberación, mientras no se alcance la depleción total del fármaco en el sistema.

\section{Modelo de Korsmeyer-Peppas}

$$
\mathrm{Mt} / M_{\infty} \times 100=\mathrm{kt}^{\mathrm{n}}
$$

Donde, $M t$ es la cantidad de fármaco liberada al tiempo t y $M \infty$ es la cantidad inicial de fármaco en las nanopartículas, $k$ es la constante de liberación y $n$ un exponente adimensional, relacionado con el mecanismo de liberación. Para partículas esféricas, un valor de $n \leq 0.43$ indica un mecanismo de difusión fickiano, si se tienen valores de $\mathrm{n}$ de 0.43 a 0.85 la difusión es no-fickiana y valores de $\mathrm{n}$ superiores $\geq 0.85$ indican que el principal mecanismo de liberación es la erosión. Para determinar el exponente $n$ se utiliza la porción de la curva donde $M t / M \alpha<0.6$ (menos del $60 \%$ liberado de la dosis inicial). El modelo semi-empírico de Peppas se emplea cuando el mecanismo de liberación a partir de formas poliméricas no es bien conocido, o cuando más de un tipo de fenómenos están involucrados.

\section{Cinética de orden cero}

$$
\mathrm{Mo}-\mathrm{Mt}=\mathrm{Kt}
$$

Donde, Mo es la cantidad inicial de fármaco en el sistema de liberación, Mt es la cantidad de fármaco en el sistema de liberación al tiempo t y $\mathrm{K}$ es una constante de proporcionalidad que representa a la constante de liberación de orden cero. En este caso se libera la misma cantidad de fármaco por unidad de tiempo, por lo que un gráfico de cantidad liberada versus tiempo es lineal, si se cumple con ciertas condiciones como que la liberación del fármaco será gradual, sin que el área del sistema de liberación sufra cambios o que el sistema se erosione.

\section{Cinética de primer orden}

$$
\ln \mathrm{Mt}=\ln \mathrm{Mo} \mathrm{K}_{1} \mathrm{t}
$$

Donde $M t$ es la cantidad de fármaco liberado al tiempo $t$, Mo es la cantidad inicial de fármaco en la solución y $K_{1}$ es la constante de liberación de primer orden. Un sistema de liberación con este perfil libera el fármaco de manera proporcional a la cantidad de fármaco remanente en el sistema, por lo que la cantidad de fármaco liberada por unidad de tiempo va disminuyendo. Por ello, el gráfico del logaritmo de la cantidad liberada versus tiempo es lineal.

Tabla 4. Valores de $\mathrm{R}^{2}$ y constantes para la regresión lineal de cada modelo

\begin{tabular}{|c|c|c|c|c|c|c|}
\hline Formulación & \multicolumn{2}{|c|}{ Modelo Peppas } & \multicolumn{2}{c|}{ Modelo Higuchi } & Cinética de orden 0 & Cinética de 1er orden \\
\hline & $R^{2}$ & $n$ & $R^{2}$ & $k H$ & $R^{2}$ & $R^{2}$ \\
\hline Ps-20MCP & 0,9948 & 1,8046 & 0,9922 & 1,0815 & 0,9769 & 0,9855 \\
\hline Ps-30MCP & 0,9839 & 0,9189 & 0,9968 & 1,4269 & 0,9780 & 0,9921 \\
\hline
\end{tabular}

Los resultados mostrados en la Tabla 4, revelan que ambas formulaciones se ajustan al modelo de Higuchi y exhiben una cinética de primer orden. Lo cual permite asumir que la difusión es el principal mecanismo de liberación, es decir el fármaco difunde a través del polímero y la velocidad con que se libera depende de la concentración del fármaco (Tas et al., 2009). Este comportamiento es el que generalmente se observa para fármacos solubles en agua: la liberación a partir de la matriz que los contiene ocurre por difusión. 


\section{CONCLUSIONES}

Las nanopartículas obtenidas poseen las características tecnológicas adecuadas en cuanto a tamaño y contenido de fármaco, por lo cual se consideran potencialmente adecuadas para la administración nasal de metoclopramida. Adicionalmente, el uso de un polímero mucoadhesivo le confiere a las partículas una ventaja para la liberación del fármaco cargado. El perfil de liberación observado, consistente de una liberación gradual del fármaco, con una liberación inicial grande y rápida (efecto ráfaga), en las primeras horas posteriores a su administración, permite suponer que se logrará llegar rápidamente a la concentración plasmática que promueva el efecto terapéutico deseado.

\section{AGRADECIMIENTOS}

Los autores agradecen a Moléculas Finas de México por la donación de metoclopramida $\mathrm{HCl}$ y a Ashland Specialty Ingredients por la generosa donación de Gantrez AN 119®. De igual manera al proyecto PAPIIT IN216016 (UNAM) y PIAPI 1803 (UNAM). Agradecen igualmente al Departamento Administrativo de Ciencia, Tecnología e Innovación - COLCIENCIAS en Colombia por su apoyo.

\section{REFERENCIAS}

Aguilar-Rosas, I., Alcalá-Alcalá, S., Llera-Rojas, V. y Ganem-Rondero, A., Preparation and Characterization of Mucoadhesive Nanoparticles of Poly (methyl vinyl ether-co-maleic anhydride) containing Glycyrrhizic Acid Intended for Vaginal Administration, http://dx.doi.org/10.3109/03639045.2014.980425, Drug Dev Ind Pharm, 41(10), 1632-1669 (2014)

Arbós, P., Wirth, M. y otros tres autores, Gantrez $z^{\circledR} A N$ as a new Polymer for the Preparation of Ligand-Nanoparticle Conjugates, J. Control. Release, 83(3), 321-330 (2002)

Armbruster, D. A., Pry, T., Limit of blank, limit of detection and limit of quantitation, Clin Biochem Rev, 29(1), 49-52 (2008)

Basante-Romo, M., Gutiérrez, O. y Camargo-Amado, R.J., Evaluación de la Citotoxicidad Inducida por TiO $\mathrm{Modificado}$ Funcionalizado con Folato y Oro sobre Líneas Celulares de HeLa Y CHO, http://dx.doi.org/10.4067/S071807642016000500008, Inf. Tecnol, 27(5), 63-68 (2016)

Butani, D., Yewale, C. y Misra, A., Topical Amphotericin B Solid Lipid Nanoparticles: Design and Development, https://doi.org/10.1016/j.colsurfb.2015.07.032, Colloids Surf. B, 139, 17-24 (2016)

Camacho, A.I., Da Costa Martins, R. y otros siete autores, Poly(methyl vinyl ether-co-maleic anhydride) Nanoparticles as Innate Immune System Activators, https://doi.org/10.1016/j.vaccine.2011.05.072, Vaccine, 29(41), 7130-7135 (2011)

Cerchiara, T., Luppi, B. y otros tres autores, Chitosan and Poly(methyl vinyl ether-co-maleic anhydride) Microparticles as Nasal Sustained Delivery Systems, https://doi.org/10.1016/j.ejpb.2005.05.005, Eur. J. Pharm. Biopharm., 61(3), 195-200 (2005)

Chen, A.Z., Wang, G.Y. y otros cuatro autores. Preparation of Poly-(methyl vinyl ether-co-maleic anhydride) Nanoparticles by Solution-Enhanced Dispersion by Supercritical $\mathrm{CO}_{2}$, https://doi.org/10.3390/ma5101841, Materials, 5(10), 1841-1852 (2012)

Chen, Y., Yang, X. y otros cinco autores, Preparation and Characterization of a Nanostructured Lipid Carrier for a Poorly Soluble Drug, https://doi.org/10.1016/j.colsurfa.2014.04.032, Colloids Surf. A, 455(1), 36-43 (2014)

Chung, K.H., Wu, C.S. y Malawer, E.G., Glass transition Temperatures of Poly(methyl vinyl ether-co-maleic anhydride) (PMVEMA) and Poly(methyl vinyl ether-co-maleic acid) (PMVEMAC) and the Kinetics of Dehydration of PMVEMAC by Thermal Analysis, https://doi.org/10.1002/app.1990.070410326, J. Appl, Polym. Sci., 41(34), 793-803 (1990)

Costa, P. y Sousa Lobo, J.M., Modeling and comparison of dissolution profiles, https://doi.org/10.1016/S09280987(01)00095-1, Eur. J. Pharm., 13(2), 123-133 (2001)

Crucho, C.I.C. y Barros, M.T., Polymeric nanoparticles: A Study on the Preparation Variables and Characterization Methods, https://doi.org/10.1016/j.msec.2017.06.004, Mater. Sci. Eng. C, 80, 771-784 (2017)

Delgado, A.E., Palacio, O. y Aperador, W., Efecto de Butil Hidroxitolueno (BHT) en la Estabilidad Oxidativa de un Lubricante a Base de Aceite de Ajonjolí. http://dx.doi.org/10.4067/S0718-07642015000400011, Inf. Tecnol, 26(4), 81-88 (2015)

El-Habeeb, A.A., Al-Saif, F.A. y Refat, M.S., Charge-Transfer Interactions of Metoclopramide Nausea Drug Against Six Kind of p-acceptors: Spectral and Thermal Discussions, https://doi.org/10.1016/j.saa.2013.12.093, Spectrochim Acta A Mol Biomol Spectrosc, 123, 455-466 (2014)

Flores, J. E., Villaflor, G. J., Velasco, W. y Bisonard, E. R., Determinación de la Velocidad de Transporte de Partículas por Saltos en Conductos Horizontales, http://dx.doi.org/10.4067/S0718-07642017000600021, Inf. Tecnol., 28(6), 199-106 (2017)

González, R. E, Tarón, A. y Pérez, J., Biopelículas de Gelana Cargadas con Nanopartículas de Plata: Propiedades Físico Mecánicas, Antimicrobianas y de Barrera. http://dx.doi.org/10.4067/S0718-07642017000600007, Inf. Tecnol., 28(6), 5360 (2017) 
Illum, L., Nasal Drug Delivery: New Developments and Strategies, https://doi.org/10.1016/S1359-6446(02)02529-1, Drug Discov. Today, 7(23), 1184-1189 (2002)

Irache, J. M., Huici, M. y otros cuatro autores. Bioadhesive properties of Gantrez nanoparticles. https://doi.org/10.3390/10010126, Molecules, 10(1), 126-145. (2005)

Kumari, A., Yadav, S.A. y Yadav, S.C., Biodegradable Polymeric Nanoparticles Based Drug Delivery Systems, https://doi.org/10.1016/j.colsurfb.2009.09.001, Colloids Surf. B, 75(1), 1-18 (2010)

López, J. M., Ensuncho, A.E. y Robles, J., Estudio Teórico de la Reactividad Química y Biológica de Cisplatino y algunos Derivados con Actividad Anticancerosa, http://dx.doi.org/10.4067/S0718-07642013000300002, Inf. Tecnol, 24(3), 3-14 (2013)

Petropoulos, J. H., Papadokostaki, K. G. y Sanopoulou, M., Higuchi's equation and beyond: Overview of the formulation and application of a generalized model of drug release from polymeric matrices, https://doi.org/10.1016/j.ijpharm.2012.08.012, Int. J. Pharm. 437(1-2), 178-191 (2012)

Pires, P.C. y Santos, A.O., Nanosystems in Nose-to-Brain Drug Delivery: A Review of Non-Clinical Brain Targeting Studies, https://doi.org/10.1016/j.jconrel.2017.11.047, J. Control. Release, 270, 89-100 (2018)

Rezaeinia, H., Ghorani, B., Emadzadeh, B. y Tucker, N., Electrohydrodynamic atomization of Balangu (Lallemantia royleana) seed gum for the fast-release of Mentha longifolia L. essential oil: Characterization of nano-capsules and modeling the kinetics of release, https://doi.org/10.1016/j.foodhyd.2019.02.018, Food Hydrocoll., 93(October 2018), 374385 (2019)

Salade, L., Wauthoz, N., Goole, J. y Amighi, K., How to Characterize a Nasal Product. The State of The Art of In Vitro and Ex Vivo Specific Methods, https://doi.org/10.1016/j.ijpharm.2019.02.026, Int. J. Pharm., 561, 47-65 (2019)

Salinas-Salas, G. E, y Espinosa, F. F., Construcción y Calibración de una Celda para la Determinación de la Distribución de Tamaño de Micro Partículas. http://dx.doi.org/10.4067/S0718-07642011000300010, Inf. Tecnol, 22(3), 79-88 (2011)

Sosnik, A., Das Neves, J. y Sarmento, B., Mucoadhesive Polymers in The Design of Nano-Drug Delivery Systems for Administration By Non-Parenteral Routes: A Review, https://doi.org/10.1016/j.progpolymsci.2014.07.010, Prog. Polym. Sci., 39(12), 2030-2075 (2014)

Tas, C., Ozkan, C.K., y otros cuatro autores. Nasal Administration of Metoclopramide from Different Dosage Forms: In Vitro, Ex Vivo, and In Vivo Evaluation, https://doi.org/10.1080/10717540902764172, Drug Deliv., 16(3), 167-175 (2009)

Valencia, S. H., Marín, J. M. y Restrepo, G. M., Efecto del pH en la Degradación Fotocatalítica de Materia Orgánica Natural. http://dx.doi.org/10.4067/S0718-07642011000500008, Inf. Tecnol, 22(5), 57-66 (2011)

Wu, I. Y., Bala, S., Škalko-Basnet, N. y di Cagno, M. P., Interpreting non-linear drug diffusion data: Utilizing KorsmeyerPeppas model to study drug release from liposomes. https://doi.org/10.1016/j.ejps.2019.105026, Eur. J. Pharm. 138(June), 105026 (2019)

Zaki, N.M., Awad, G.A., Mortada, N.D. y Abd ElHady, S.S., Enhanced Bioavailability of Metoclopramide HCl by Intranasal Administration of a Mucoadhesive In Situ Gel with Modulated Rheological and Mucociliary Transport Properties, https://doi.org/10.1016/j.ejps.2007.08.006, Eur. J. Pharm. Sci., 32(4-5), 296-307 (2007) 